\title{
Some important features of the proposed new definition of the International System of Units (SI): realization and hierarchical problems that the users should know about
}

\author{
Franco Pavese ${ }^{1, *}$ and Abdérafi Charki ${ }^{2}$ \\ ${ }^{1}$ Formerly at the National Research Council of Italy, IMGC-CNR, Torino, Italy \\ ${ }^{2}$ University of Angers, 62 av. Notre Dame du Lac, 49000 Angers, France
}

Received: 3 October 2016 / Accepted: 11 October 2016

\begin{abstract}
The paper, after a short introduction to measurement units in general and to the present international system of units (SI: Système International des Unités), deals with a digest of the basics of the proposed new SI:2018 and of its differences compared with the present SI. The latter issue also involves a review of some problems still unresolved in the last draft, concerning: the role of the constants of physics in the system and their role in the conceptual construction of this international standard; the method employed for using the relevant experimental data, and related statistical issues; finally, the implications for science of the New SI implementation. The consequences for and new duties of the national metrology institutes and practitioners are illustrated, involving the future conformity of the present standards and a possible hierarchy between countries that would result from the new definition, in contrast with certain principles of the Convention du Mètre.
\end{abstract}

Keywords: measurement units / standards / New SI / fundamental constants / magnitude continuity / standards conformity / hierarchy between countries

\section{Short historical introduction}

Even at the dawn of civilisation, "quantitative measurements" were performed, e.g., for the volume of goods like grains, milk, fermented drinks, etc.; for the extension of lands, fields, homes, etc.; for the determination of time. Subsequently, they evolved and a so-called "standard" of each required quantity was deemed necessary to offer the possibility of indicating by how many times a measured quantity was larger, heavier, lengthier, etc., than the relevant standard (for time measurements it was more a matter of time elapsing or differences in time). Numbers were often, but not always, used to quantify these indications.

However, a "standard" was a community-specific device, not always recognised by other communities, with obvious problems, especially for commerce. Large communities or empires thus tended to have unique standards, reproduced throughout the community. This introduced the need to know the precision needed to reproduce copies of the standards in different places according to the required purpose: for example, weighing gold or grains obviously calls for different degrees of precision.

The above need extended to techniques (the so-called "arts") where the standard most often became a "standard object". This happened before the term "science" appeared: the objects were used by scholars practising both

* Corresponding author: frpavese@gmail.com speculative and empirical activities, the latter requiring measurements, which started to require a precision level possibly preceding the practical needs.

When the importance of science became universally recognised and appreciated, the need for measurements boomed, and the preference for 'universal' standards with it.

There was a special standard for each kind of quantity, namely that to which the number 1 of magnitude was assigned: one unit of the quantity in question.

Since units of quantitative measurement are needed in almost any human activity, it is important that all scientists and practitioners have correct information and understanding of them.

Systems of units were built and eventually systems recognised worldwide - never universally, however: even the Système International des Unités (SI), the most modern of all, has not been adopted by all countries in the world (think, for example, of the British 'Imperial' units), but only by those countries having signed the Metre Convention, established 150 years ago, originally by a consortium of 17 nations. The signatories today number 57: for a historical overview one can consult the BIPM website [1]. The Convention set up a series of Bodies for its regulation, and a Bureau International des Poids et Mesures (BIPM), with headquarters in an extraterritorial enclave in Sèvres, near Paris.

The SI is a system that has evolved with time, as shown in [1].

The original definition of the SI can be found on the website of the BIPM, under "Metre Convention" [2] and under "SI Brochure" [3]: 
"The Convention was signed in Paris in 1875 by representatives of seventeen nations. ... the Metre Convention established a permanent organizational structure for member governments to act in common accord on all matters relating to units of measurement. The Convention ... remains the basis of international agreement on units of measurement. The BIPM now has fifty-seven Member States, including all the major industrialized countries." [2].

"This SI consists of a set of base units, prefixes and derived units, as described in these pages. The SI base units are a choice of seven well-defined units which by convention are regarded as dimensionally independent: the metre, the kilogram, the second, the ampere, the kelvin, the mole, and the candela. Derived units are formed by combining the base units according to the algebraic relations linking the corresponding quantities." [3].

"Les unités choisies doivent être accessibles à tous, supposées constantes dans le temps et l'espace, et faciles à réaliser avec une exactitude élevée" (from the official French text) [3].

For the complex matter that is its subject, this paper is structured as follows: Section 2 illustrates the features required for a modern international system of units, with reference to the implementation in the present SI. Then Section 3 summarises the basic features of the proposed New SI according to the last draft available [4]. Here ends the descriptive part of the paper.

In what follows, the intention of the paper is to highlight in Section 4 the main advantages of the New SI and in Section 5 the main residual criticalities still under discussion, by means of a critical analysis of the definitions of the units as well as of a number of issues of conformity and hierarchy in the current and future standards, respectively.

The reader can refer to an ample bibliography, including free downloadable recent literature from the BIPM website, arXiv or [1-5].

\section{Mandatory features of a modern system of units, and the present SI}

A sound system of units of modern conception must fulfil specific requirements, some of which are explicitly indicated in the above citations $[2,3]$. The units should be:

- constants in time and space;

- organised in a rational system;

- easy to realise with high precision;

- accessible to everybody.

\subsection{Constancy of units in time and space}

The constancy in space is desirable, to avoid the risk of the magnitude (see term 1.1 in [6]) of the units not being valid everywhere in the earth or universe.

The constancy in time is the most crucial feature of the units, because any change would entail, in general, problems that are difficult to solve satisfactorily for the generality of users. The failure to satisfy this condition would produce the need for conversion factors of numerical values between the old (e.g., the CGS) and new systems - not to mention a change of name to avoid confusion. In fact, every scientist or practitioner knows how many conversion factors between units exist (e.g., consult [7]) and how easy it is to miss something and introduce errors in the conversion; and consequently how difficult it is to compare results obtained using different systems of units.

A measurement unit must have a numerical value in its definition to fix the exact measure of magnitude intended for that 'kind of quantity' (see term 1.2 in [6]). This is required because a unit has traditionally to be univocalirrespective of the precision of its realization in practice.

The way by which this property applies may vary depending on the nature of the unit and on the required type of measurement scale.

For example, let us consider first the case of an artefact materializing a specific unit (type (a) standard in Section 2.2 and Type 1 in $[8,9]$ ), which at present is the case for the unit of mass and of the object that is assigned the precise numerical value of $1 \mathrm{~kg}$, the so-called IPK of BIPM: it is the 'realization' of the present-SI mass unit used in measurement.

One has to make a clear distinction between the uniqueness of this type of measurand (its definition being univocal) and possible physical changes to such an object: the former may be time independent; the latter can only be time dependent. The latter is mainly due to wear during use, and consequently is the object of studies and international concern; the former means in this case that the (solid) material object was perfectly defined.

However, in modern times, questions have arisen about the definition of the interface between the artefact and the environment: in fact, in the case of mass measurements, a vast literature exists about the pros and cons of performing measurements in vacuum, where it is assumed that, in principle, the interface should be more precisely defined. In the same field of mass standards, similar studies, much more refined, have been possible in the case of the "Avogadro Project", where the artefact is a silicon object of spherical shape.

The non-uniqueness in these cases comes from differences in composition of the solid mass causing local variability in density; and, of thickness of the adherent interface layer(s), having an effect different from zero on the actual dimensions of the object, i.e. adding additional mass to the object with possibly timedependent variability.

Also in the case of the first length standard, a graduated metal bar, a problem of non-uniqueness arose when the width and shape of the graduations engraved on the bar introduced non-uniqueness in the measurement of distances between graduations.

In both cases, the stipulation of an exact value (conventional value) was the only way to remove the non-uniqueness. This entails the need for a distinction between the exact conventional value and the result of any experimental realization of it-determination of the magnitude - that is affected by an uncertainty and needs data analysis. 
Considering now, instead, units defined by means of physical-chemical 'states' or 'parameters' or 'conditions' (type (b) standards in Section 2.2 and Type 2 in $[8,9]$ ), let us consider the unit for temperature.

This case is additionally different from the previous ones discussed because the former concerned "extensive" quantities and the corresponding types of measurement scale, while temperature is an "intensive" quantity requiring a different type of scale (the terms extensive and intensive sometimes today being considered outdated) [10].

Limiting the illustration here to the non-uniqueness of the quantity, the intention in this case is to take advantage of a state of the matter that should have sufficient theoretical bases to ideally be considered unique. In the case of the temperature scale with the unit kelvin, such a state is presently the "triple point" of a specific substance, water.

Is it true, though? Term 2.11 in [6] indicates in Note 1: " . . . owing to the inherently incomplete amount of detail in the definition of a quantity, there is not a single true quantity value but rather a set of true quantity values consistent with the definition". This means that, with the refinement of the uncertainty of the realizations, irrelevant details (properties) of a substance may become relevant, inducing non-uniqueness. For example, in the case of water, over the last couple of decades the specification of a conventional isotopic composition has been deemed necessary - formalised in 2005 [11].

The need for additional "details" in a definition also concerns the unit of time, where, for the second, the frequency of "the radiation corresponding to the transition between the two hyperfine levels of the ground state of the caesium 133 atom" [12] was chosen, however later specifying that "this definition refers to a caesium atom at rest at a temperature of $0 \mathrm{~K}$ " and, further, that "it is understood that the $C$ s atom at a temperature of $T=0 \mathrm{~K}$ is unperturbed by black-body radiation".

In general, this was the fate of all units based on these types of definition: an affordable but undesirable need for evolution in time of those definitions to avoid significant non-uniqueness, not necessarily requiring change to the stipulated numerical value, simply attributed to the stipulated state.

The above facts were the principal reasons behind the idea of using more constants independent of any substance of physics and chemistry. Sometimes the need emerges instead of a brand-new definition of some, or all, units [1]. However, there is one very specific requirement of a system of units: implicitly a new definition must ensure the continuity of the magnitude with the previous definition of the same unit - by some authors called "constancy" in time of the unit (e.g., see [13]).

\subsection{Rational system of units}

A system of units must be built according to a robust conceptual frame, so that there are not inconsistencies (either conceptual or factual) in its structure or in the set of numerical values used in the definition of the units.
This frame begins with the definition of the basic characteristics of the system: these are the quantities comprised in the system - generally a subset of the general set of all types of quantities that may require to be measured, excluding so far all the non-quantitative measurementsand how they are named and related to each other.

Concerning how they are related to each other, it is desirable that the system be of the type called "coherent", in other words that in the algebraic relations between the units no numerical factors are needed. The present SI is a coherent system for the set of "base quantities".

The setting of "base quantities" is another way to interrelate different quantities and their units: for the present SI, as spelled out in the text in [3] reported in Section 2, this means that all the units outside the set of base units are called "derived" and are "formed by combining the base units according to the algebraic relations linking the corresponding quantities". In the initial SI (1889, MKS system), the base units were three, namely length, mass and time; then there were four (1946, MKSA system, or Giorgi system) with the addition of the unit for electrical current. Currently, and this since 1948, there are seven, with the addition of the units for temperature, amount of substance and luminous intensity.

Up to that stage of the conceptual construction, no numerical values need be indicated.

However, from then on the definition of any unit requires the specification of at least one numerical value: this indicates the condition that the relevant property (e.g. length) must comply with, for the value 1 (or unitary difference) to be assigned in the proper scale. This value is deemed to be independent of realization uncertainty, irrespective of the type of data treatment.

The SI, even the present one, is built according to the principles indicated. The definition of the system presently consists of the definition of seven base units, in which a numerical indication of the magnitude of each unit is specified. In the specific jargon, it is of the type called "Explicit Unit Definition" (EUD).

Each of the base units is individually defined. The present definitions comprise different types:

(a) Make use of an artefact: e.g., for mass "The kilogram is the unit of mass; it is equal to the mass of the international prototype of the kilogram";

(b) Make use of a physical state or condition: e.g., for temperature "The kelvin, unit of thermodynamic temperature, is the fraction 1/273.16 of the thermodynamic temperature of the triple point of water". A similar approach for time, amount of substance and luminous intensity;

(c) Define a realization method of the unit, e.g. for length: "The metre is the length of the path travelled by light in vacuum during a time interval of 1/299 792 458 of a second'. With a similar approach for electrical current.

(Actually, the type (a) and (b) base units also define indirectly a so-called "realization method", to be implemented with a procedure (not to be confused with the "mise en pratique" [11] which is a (set of) approximations of the definition), according to clause 2.6 of VIM [6]). 
At present, four base units violate the indication of the French text of the SI Brochure that "les grandeurs de base sont, par convention, considérées comme indépendantes" (Section 1.2 in [3]), because, using for convenience some derived units too, the definition of: the unit of length involves the second; the unit of amount of substance involves the kilogram; the unit of electric current involves the newton and the metre; the unit of luminous intensity involves the hertz, the watt and the steradian.

This fact has important consequences on a property that is mandatory for any system of units, and consequently often assumed to be achieved by the SI: its internal consistency.

For base units defined independently of each other, consistency is automatically, i.e. intrinsically, achieved: the numerical values, i.e. their magnitudes, can be assigned arbitrarily.

For base units dimensionally inter-dependent, consistency cannot be assumed, nor can it be precisely implemented. For non-independent definitions, one can only verify formal or logical consistency, the latter in the sense of a syntactic property relating unit definitions, but this can only be proved to a certain 'degree' and can be somewhat problematic.

There is another, more specifically metrological, meaning of the term consistency that is often used. It is defined in [6] as a kind of "metrological compatibility", a property of a set of experimental determinations affected by uncertainty. For details see [14].

\subsection{Easy high-precision implementation}

This is a basic requirement for all users, from practitioners to top metrologists, because this system is assumed to be used in any country at any level of precision.

Metrology - and thus the implementation and use of a system of units - is a hierarchical frame with scientific, technical, but also legal aspects. This is because the system must assure the traceability of the measurements, and consequently of the standards. In VIM [6] "metrological traceability" is defined as the "property of a measurement result whereby the result can be related to a reference through a documented unbroken chain of calibrations, each contributing to the measurement uncertainty", implying that "metrological traceability requires an established calibration hierarchy", as stated in Note 2 of clause 2.41. Consequently, only those laboratories and users can sit at the top of the hierarchy who can demonstrate having realised the definitions of the relevant measurement units.

In the case of the present SI, this means having implemented the "definitional method" included in the definition of each unit. The standards of those not having implemented them, in any other country, therefore, will necessarily depend on the calibration of local national standards against one of the standards of another country which has achieved that goal. As a consequence of this fact, the former stay at a lower step of the international hierarchical metrological ladder, with the latter at the top. However, this hierarchy applies only on free decision of each single country and only for specific units.

\subsection{The system of units should be accessible to everybody}

All the signatory countries of the Metre Convention are supposed to benefit equally from access to the whole of the Convention. This means that, in principle, all the National Metrological Institutes (NMI), which are at the top of their national metrological hierarchies, should be able, if they so wish, to get to the top of the international metrological ladder for the units of their interest. It is not a matter of precision, because each NMI in its country is at the peak of national traceability, irrespective of the precision it supplies: it only has to fit national needs. On the other hand, accessibility means that, should any country decide to raise the ultimate precision of its standards to the highest international state-of-the-art level, the system of units should facilitate that task, with a reasonable limitation of the complexity required and the resources necessary to reach that goal.

In the case of the present SI, the definitional methods have demonstrated sufficient easiness. However, in some cases, the implementation of the definitional methods can be a serious limitation, especially when considering that new methods may have been developed in the meantime, often superior to the definitional one.

The addition of a mise en pratique [15] for each base unit was intended to extend the flexibility of the NMIs in realising the standards. However, though already a useful development, it is restricted to a lower-rank status by the current base unit definitions (see Tab. 1) [16,17].

\section{Summary of the SI:2018}

The proposed change of definition is usually named "New SI", or "revised SI". Its current deadline for promulgation is set at 2018. The use only of 'constants' in the definition has been proposed - with stipulated numerical values.

\subsection{Definition of the SI:2018}

The following "Definition of the SI" is reported in the last available draft [4]:

"The International System of Units, the SI, is the system of units in which:

- the unperturbed ground state hyperfine splitting frequency of the caesium 133 atom $\Delta v_{\mathrm{Cs}}$ is $9192631770 \mathrm{~Hz}$,

- the speed of light in vacuum c is $299792458 \mathrm{~m} / \mathrm{s}$,

- the Planck constant $h$ is $6.626070040 \times 10^{-34} \mathrm{~J} \mathrm{~s}$,

- the elementary charge $e$ is $1.6021766208 \times 10^{-19} \mathrm{C}$, (1)

- the Boltzmann constant $k$ is $1.38064852 \times 10^{-23} \mathrm{~J} / \mathrm{K}$,

- the Avogadro constant $N_{A}$ is $6.022140857 \times 10^{23} \mathrm{~mol}^{-1}$,

- the luminous efficacy $K_{\mathrm{cd}}$ of monochromatic radiation of frequency $540 \times 10^{12} \mathrm{~Hz}$ is $683 \mathrm{~lm} / \mathrm{W}$.

The numerical values of the seven defining constants have no uncertainty".

The constants (no longer labelled "fundamental" since 2015) are of five different types:

- for time a specific electronic transition $\Delta \nu_{\mathrm{Cs}}$ of a specific substance, ${ }^{133} \mathrm{Cs}$ - the same as used at present; 
Table 1. Metrological ladder for the SI (example: length). ${ }^{\text {a }}$

\begin{tabular}{lll}
\hline Traceability & Today & New SI \\
\hline Top & $\begin{array}{l}\text { Definitional method. } \\
\text { Method using "distance" and "time interval" }\end{array}$ & $\begin{array}{l}\text { No definitional method. } \\
\text { Condition: to reproduce the stipulated constant(s) value } \\
\text { "Primary" methods. }\end{array}$ \\
& $\begin{array}{l}\text { Mise en pratique. } \\
\text { Other method(s): } \\
\text { frequency and period }\end{array}$ & $c_{0}$ and $t$ explicitly in the model \\
-2 & Secondary methods. & Mise en pratique. \\
& Other method(s): & Other method(s): frequency and period \\
-3 & stabilised laser & \\
& Workshop methods. & Secondary methods. \\
& Gauge blocks & Other method(s): \\
& Lower ranks & stabilised laser \\
$\ldots$ & $\ldots$ & Workshop methods: \\
\hline
\end{tabular}

a See term in [6].

- for luminous intensity, a technical constant $K_{\mathrm{cd}}$, of physiological origin - the same as used at present;

- for electrical current, the elementary charge $e$, via the fine-structure constant $\alpha=e^{2} /\left(2 c \varepsilon_{0} h\right)$;

- for temperature and amount of substance, the Boltzmann constant $k$ and the Avogadro constant $N_{\mathrm{A}}$, respectively, considered two 'conversion factors';

- for length and mass, the speed of light in vacuum $c$ - the same as at present - and the Planck constant $h$, respectively, the only two real "fundamental" constants.

This can be called the 'group-definition' - in jargon, it is of the type called "Explicit Constant Definition" (ECD) or "Global Constant Definition" (GCD), since these constants should be considered and treated as a group.

It is indicated as a sufficient new SI definition.

Table 1 in [4] indicates that these constants define the following units: $\mathrm{Hz}=\mathrm{s}^{-1}, \mathrm{~m} \mathrm{~s}^{-1}, \mathrm{Js}=\mathrm{kg} \mathrm{m}^{2} \mathrm{~s}^{-1}, \mathrm{C}=\mathrm{As}$, $\mathrm{J} \mathrm{K}^{-1}, \mathrm{~mol}^{-1}, \operatorname{lm} \mathrm{W}^{-1}=\mathrm{cdsr} \mathrm{W}^{-1}$.

\subsection{Definitions of the SI:2018 units: base units}

Additionally to the group definition of the constants, in [18] it was also stated that: "The SI may alternatively be defined by statements that explicitly define seven individual base units: the second, metre, kilogram, ampere, kelvin, mole, and candela. These correspond to the seven base quantities time, length, mass, electric current, thermodynamic temperature, amount of substance, and luminous intensity. All other units are then obtained as products of powers of the seven base units, which involve no numerical factors; these are called coherent derived units".

However, in the more recent draft [4], the position is different: the "description in terms of base and derived units is maintained in the present definition of the SI, but has been reformulated as a consequence of adoption of the defining constants". The reason for this can be found in the statement that "Preserving continuity is an essential feature of any changes to the International System of Units". Moreover, "the definitions of the traditional base units of the SI ... follow from the definition of the seven defining constants".
Therefore, the definition for each single base unit is also provided. At present, the above statement is implemented in [4] for each of the seven base units as follows:

- "The second, symbols, is the SI unit of time. It is defined by taking the fixed numerical value of the caesium frequency $\Delta v_{\mathrm{Cs}}$, the unperturbed ground-state hyperfine splitting frequency of the caesium 133 atom, to be 9192631770 when expressed in the unit $\mathrm{Hz}$, which is equal to $\mathrm{s}^{-1}$ for periodic phenomena."

- "The mole, symbol mol, is the SI unit of amount of substance of a specified elementary entity, which may be an atom, molecule, ion, electron, any other particle or a specified group of such particles. It is defined by taking the fixed numerical value of the Avogadro constant $N_{\mathrm{A}}$ to be $6.022140857 \times 10^{23}$ when expressed in the unit $\mathrm{mol}^{-1}$."

- "The metre, symbolm, is the SI unit of length. It is defined by taking the fixed numerical value of the speed of light in vacuum $c$ to be 299792458 when expressed in the unit $\mathrm{ms}^{-1}$, where the second is defined in terms of the caesium frequency $\Delta v_{\mathrm{Cs}}$."

- "The kilogram, symbol $\mathrm{kg}$, is the SI unit of mass. It is defined by taking the fixed numerical value of the Planck constant $h$ to be $6.626070040 \times 10^{-34}$ when expressed in the unit $\mathrm{J} \mathrm{s}$, which is equal to $\mathrm{kg} \mathrm{m}^{2} \mathrm{~s}^{-1}$, where the metre and the second are defined in terms of $c$ and $\Delta v_{\mathrm{Cs}}$."

- "The ampere, symbol A, is the SI unit of electric current. It is defined by taking the fixed numerical value of the elementary charge e to be $1.6021766208 \times 10^{-19}$ when expressed in the unit $\mathrm{C}$, which is equal to $\mathrm{As}$, where the second is defined in terms of $\Delta v_{\mathrm{Cs}}$."

- "The kelvin, symbol K, is the SI unit of thermodynamic temperature. It is defined by taking the fixed numerical value of the Boltzmann constant $k$ to be $1.38064852 \times 10^{-23}$ when expressed in the unit $\mathrm{JK}^{-1}$, which is equal to $\mathrm{kg} \mathrm{m}^{2} \mathrm{~s}^{-2} \mathrm{~K}^{-1}$, where the kilogram, metre and second are defined in terms of $h$, $c$ and $\Delta v_{\mathrm{Cs}}$." - "The candela, symbol cd, is the SI unit of luminous intensity in a given direction. It is defined by taking the fixed numerical value of the luminous efficacy of monochromatic radiation of frequency $540 \times 10^{12} \mathrm{~Hz}$, 
$K_{\mathrm{cd}}$, to be 683 when expressed in the unit $1 \mathrm{~m} \mathrm{~W}^{-1}$, which is equal to $\mathrm{cd} \mathrm{sr} \mathrm{W}^{-1}$, or $\mathrm{kg}^{-1} \mathrm{~m}^{-2} \mathrm{~s}^{3} \mathrm{cdsr}$, where the kilogram, metre and second are defined in terms of $h$, $c$ and $\Delta v_{\mathrm{Cs}}$ ".

The above definitions imply that the present base units - second, metre, kilogram, ampere, kelvin, mole, and candela-remain in the "New SI", however with totally differently-based definitions and function: they are no longer the definition of the SI (see Sect. 5.1).

They are maintained in order to fulfil the "continuity condition" and are indicated to "follow" from the SI definition - the 'group' definition of the constants. In all instances, some units remain undefined even in the above definitions: $\mathrm{Hz}, \mathrm{J}, \mathrm{lm}, \mathrm{W}$, and $\mathrm{sr}$ are defined as derived units only later in the conceptual roadmap.

\section{Basic features of the SI:2018}

The proposal of the "New SI" to anchor the magnitude of the base units to constants entails some basic new features that are the pillars of the new definition and can be considered an interesting improvement with respect to the present SI. They are summarised here.

Internal consistency of the base-unit system. Only a degree of consistency can be checked, because four of the seven constants $\left(c\right.$ (also called $\left.c_{0}\right), h, e, k$ (also called $\left.\left.k_{\mathrm{B}}\right)\right)$ are multi-dimensional, so an arbitrary choice of unit magnitudes is not allowed. For stipulation, the present values of the seven constants were obtained by making use of the CODATA studies [19], which fix the (minimum) degree of consistency that is acceptable for the SI from now on. This is an important asset of the proposal. See end of Section 2.2 for more on this issue.

Continuity (constancy) of the unit magnitude. The continuity requirement (also called constancy in time of the unit magnitudes) is stressed in the proposal as a basic need. Because of this requirement, the set of basic units is taken unaltered from the present SI, but only as to the base quantities and unit denominations, while the definition of the base units is now founded on the same constants as the group definition. In addition, since the stipulated values of the constants arise from the present-SI, the magnitudes of those constants are assumed to remain unaltered. See Section 2.1 for more on this issue.

Actually, only the use of three of the seven constants is new: $h, k$ and $e$. In fact, $\Delta v_{\mathrm{Cs}}, c_{0}, N_{\mathrm{A}}$ and $K_{\mathrm{cd}}$ are already included in the present-SI definitions.

Lack of definitional methods. In the New SI there is no longer a definitional method specified for each base unit. This is perhaps the most important practical change in the features of the SI of interest to top-level practitioners. This issue is illustrated in Table 1, which compares the present and the future situations: see Section 5.2.1 for more on this issue. It means that "the realizations are separated conceptually from the definitions" [4].

In addition, to be on the top step of the hierarchical ladder, an institute should now demonstrate that its standards comply with the new condition set by SI:2018: that, by using them, it is able to be consistent with the stipulated value of each relevant constant - in the past it had only to demonstrate that it possessed consistent realizations of the relevant "definitional methods".

Lack of reference to any specific substance: uniqueness. The preference for use of constants in the strict sensethough of different types, see Section 3.1-is born of the fact that they are not a property of any specific substance. Only for time, whose definition is formulated differently from the present one but is basically the same, the atomic transition is still one of ${ }^{133} \mathrm{Cs}$. The advantage is that of a unique property of the constants: their lack of definitional non-uniqueness - see Section 2.1 for more on this issue.

Consequently, the "value of a constant" is the only relevant property here, which is intrinsically invariant in time and space. The numerical value, instead, is contingently assigned as the 'best' available at the time of the SI change of definition.

\section{Main criticalities in the SI:2018 2016 draft}

\subsection{Concerning unit definitions}

In this sub-section, only the main issue will be illustrated, concerning the conceptual frame of the new definition: it is based on an analysis that can be found in [20,21].

The SI-2018, by contrast with the present one, is structured into two conceptual 'frames', which will be called here A (constants) and B (base units), and which can be found in the text of the 2016 Draft [4] in the following order.

General principles

(A1) Certain advantages recognised in the present SI have "led to the decision to define all units with the help of defining constants";

(B2) The "description in terms of base and derived units is maintained in the present definition of the SI, but has been reformulated as a consequence of adoption of the defining constants";

"Definition of the $S I$ "

(A3) For 'group-definition', see Section 3.1. For each constant, it is of the type: "the [constant] [symbol] is [numerical value] [present-SI units]", where the indicated units, single or algebraic combination, can be formed by either present base units or derived units. For example, "the Planck constant h is $6.626070040 \times 10^{-34} \mathrm{~J} \mathrm{s";}$

(A4) Table 1 in [4] has the caption "The seven defining constants of the SI, and the seven corresponding units they define", where the name, symbol, numerical value and "Unit" are given, for example for $h$ is $\mathrm{J} \mathrm{s}=\mathrm{kg} \mathrm{m}^{2} \mathrm{~s}^{-1}$, where the initial units are then spelled out in terms of only the present base units, except for the luminous efficacy $K_{\mathrm{cd}}$, which is $\operatorname{lm} \mathrm{W}^{-1}=\mathrm{cdsr} \mathrm{W}^{-1}$, where a derived unit and a special name unit are indicated;

Definitions of the base units

(A5-B5) "Preserving continuity is an essential feature of any changes to the International System of Units, which has always been assured in all changes to the definitions. The numerical values of the defining constants have been chosen to be consistent with the earlier definitions insofar as advances in science and knowledge allow"; 
(B6) "The definitions of the traditional base units of the SI, as listed in Table 2 [4], follow from the definition of the seven defining constants";

(B7) The new definition of each of the seven present base units is provided. They are reported here in Section 3.2. For each base unit, it is of the following type: "[The base unit] is defined by taking the fixed numerical value of [one constant, different for each base unit], to be [numerical value] when expressed in the [present-SI units], where the [other present-SI base units indicated] is [or are] defined in terms of the [(other) relevant constant indicated in the group definition]". For example, "the kilogram . . . is defined by taking the fixed numerical value of the Planck constant $h$ to be $6.626070040 \times 10^{-34}$ when expressed in the unit $\mathrm{J} \mathrm{s}$, which is equal to $\mathrm{kg} \mathrm{m}^{2} \mathrm{~s}^{-1}$, where the metre and the second are defined in terms of $c$ and $\Delta v_{\mathrm{Cs}}$ ";

Definitions of the derived units

(B8) Table 4 [4], "The [derived] 22 SI units with special names and symbols": "Derived units are defined as products of powers of the base units", namely the joule $(\mathrm{J})$, hertz $(\mathrm{Hz})$, watt $(\mathrm{W})$, coulomb $(\mathrm{C})$, lumen $(\mathrm{lm})$ and steradian (sr).

The splitting of the New SI structure into two frames involves a number of difficulties as regards correct implementation, many still unresolved.

\subsubsection{Group definition (frame A)}

There is an obvious flaw in the conceptual sequence. In fact, at step A3 concerning the definition of the SI, the new definitions of the base and derived units have not yet been provided: it is improper in a definition to refer to a prior knowledge of the reader about the existence of certain previous units - statement B2 in itself is not sufficient to alleviate the problem.

It is not possible to disentangle the kind of definition found in A3 and resolve the flaw, because the reason for it is the fact that the numerical value of the constant is indicated, imposing the need to specify the units in which it is expressed. The reason for this choice cannot be found within frame A, but is due to frame B according to step B5.

Actually, the choice of the constants does not depend on frame $\mathrm{B}$, except for the fact that, according to the proposers, there must be seven of them, as with the present base units - in accordance with B5 - involving all the seven present ones, but without one-to-one relation with respect to the base units. However, considering that four of the seven are multi-dimensional one could in principle select fewer than seven constants, provided that these included all the dimensions of the base units.

From a conceptual viewpoint, the group definition does not need to - indeed, cannot - anticipate any numerical value. It should be considered as the "SI-fundamental" definition [22], and it would be sufficient to say [22]:

"The SI-fundamental is the coherent system of units in which $C_{1}$ is the fundamental-unit of frequency, $C_{2}$ is the fundamental-unit of speed and $C_{3}$ is the fundamental-unit of action, ... .", where the $C_{i}$ are the chosen constants. "In this way, according to this definition, for example, the Planck constant has a value 1 fundamental unit of action".
Actually, the seven constants do not define the (previous) units indicated at step A4, but rather, as indicated in [23], frequency, speed, action, electrical charge, heat capacity, reciprocal amount of substance and luminous intensity, which would be the true new base units.

However, due to the principle of B5, the base units cannot be changed. That principle does not arise from the underlying physical principles governing the choice of the SI-fundamental, but from a practice that is considered highly desirable in metrology (and in measurement science). The issue can be labelled "SI-conventional" [22], here frame B, which is the only frame of the present-SI. Then come the numerical values, originating from the present base units.

\subsubsection{Base units (frame B)}

The need to maintain the use of base units is not justified in B1, but in B5, where it is also clear that the magnitude of each base unit should remain unchanged by the change of definitions. This is the reason (implicit in the 2016 Draft [4]) for the use of numerical values for the constants that are simply the 'best' available at the moment of the change, i.e. originating from the present-SI, as stated in the second sentence in B5.

However, in B6 there is a logical inversion of this justification, where it is stated that their definitions, as implemented in $\mathrm{B} 7$, ". . . follow from the definition of the seven defining constants". In fact, in B7 the reported numerical value is obviously the one from before the change of the SI definition, retained in the group definition: this is again a consequence of the inversion of the conceptual order illustrated in the previous subsection.

This inconsistency also arises in B7, the text of the new base unit definitions, when the latter refer to the group definition for the definition of the other base units appearing in a particular definition: e.g., in the definition of the kilogram, which is based on the Planck constant only, unit " $\mathrm{kg} \mathrm{m}^{2} \mathrm{~s}^{-1}$, where the metre and the second are defined in terms of $c$ and $\Delta v_{\mathrm{Cs}}$ ". Here a circular reasoning occurs, because the group definition is obliged to refer to the present-SI units while reporting the numerical values.

Thus, the use of a separate "SI-conventional" indicated above [19], logically coming after frame A, the SIfundamental, would also resolve the problem in the base unit definitions [22]:

"The SI-conventional is the coherent system of units in which

- the unit of time (duration) is the second, symbol s, the unit of length is the metre, symbol m, the unit of mass is the kilogram, symbol $\mathrm{kg}, \ldots$;

- the frequency $\Delta v_{\mathrm{Cs}_{2}}$ is $k_{1} \mathrm{~s}^{-1}$, the speed $c$ is $k_{2} \mathrm{~m} \mathrm{~s}^{-1}$, the action $h$ is $k_{3} \mathrm{~kg} \mathrm{~m}^{2} \mathrm{~s}^{-2}, \ldots$;

- the numerical values are $k_{1}=\ldots \times 10^{-10}, k_{2}=\ldots \times$ $10^{-9}, k_{3}=\ldots \times 10^{-34}, \ldots "$,

where the numerical values are stipulated according to the 'best' values at the moment when the new definition comes into effect. Notice that also the base units should be seen as a group. 
In practice, the numerical values spelled out in the $C C U$ group-definition are the conversion factors from the "SIfundamental" to the "SI-conventional", the two distinct frames.

\subsubsection{What is intended for units in the SI:2018?}

The Introduction in [4] states: "The definition of the SI units is established in terms of a set of seven defining constants. From the units of these defining constants the complete system of units can be derived. These seven defining constants are the most fundamental feature of the definition of the entire system of units". The expression "the units of these defining constants" is ambiguous: does it refer to the present SI units (e.g., present-SI "velocity" for $c_{0}$ ), or to the present set of base units (e.g., present-SI "length" and "time" for $c_{0}$ ), or to a New SI unit for $c_{0}$ ?

In addition, the third sentence is a statement about the primacy of the definition of the group of constants (called the group-definition hereinabove). In fact, the sentence then states: "The specific constants have been identified as the best choice reflecting the previous definition of the SI based on seven base units and the progress in science", presenting the base units as the alternative way used by the previous SI definition.

According to this approach, Section 1.1-"Motivation for the use of defining constants to define the SI' [4]- states: "the realizations are separated conceptually from the definitions", a basic statement implying that the definition does not indicate any 'definitional method' [4] like the previous SI, where, contrastingly, in practice "the definition and the realization" were "equivalent".

The lack of 'definitional methods', indicated as the effect of using constants in the definition, looks like one of the few (truly significant) reasons for the latter choice. However, the implementation problems are actually moved, from the realization by definitional methods to the relationship between definition and realizations, and to their link to the previous SI.

\subsection{Concerning unit realizations, conformity and hierarchy issues of the current and future standards}

A significant consequence of the above criticalities and of the lack of a satisfactory conceptual frame, is that it is not clear what the consequences are for the practitioners and the users when they need to implement the definition of the SI:2018, especially at national level or at top accuracy levels, in particular as regards the role of the two frames, the "fundamental" and the "conventional" one, both separately and in relation to each other.

\subsubsection{Realization of the base unit definitions, metrological traceability and calibrations at the National Metrology Institutes under the SI:2018}

To summarise, the new "SI definition" is said to consist of the "definition" of seven constants, but it can be "described" by means of the same present-SI "base units" - differently but equivalently defined. Section 1.2 "Implementation of the SI" of the 2016 Brochure [4], states that: "The definitions of the SI units as decided by the CGPM represent the highest reference level for measurement traceability to the SI', meaning that the latter level has to be that of definition of the constants.

It follows that, to get "the highest reference level for measurement traceability", any NMI should demonstrate that it has its own reference to the relevant constants. Only this equates with having realised the definition of the SI.

By contrast with the realization of the present-SI, in the New SI the NMIs no longer have the task of realising a definitional method stated or implied by each unit definition - because the New SI does not have such a category of methods. They had so far instead to perform a realization of the definition of the constants. That means that they had so far to demonstrate that they can assume, as the correct numerical values of those constants, the stipulated ones.

A variety of methods exist to this effect, which can also change in time: "It is not limited by today's science or technology but future developments may lead to different ways of realizing units to higher accuracy. Defined in this way, there is, in principle, no limit to the accuracy with which a unit might be realized" [4].

However, these statements are taken from the second sentence of Section 2.2.2 of [4] entitled "Practical realization of SI units". The word "practical" looks misused, or at least ambiguous and unnecessary, as any user reading the first sentence of Section 2.2.2 of [4] can easily understand: "The highest-level experimental methods used for the realization of units using the equations of physics are known as primary methods. The essential characteristic of a primary method is that it allows a quantity to be measured in a particular unit by using only measurements of quantities that do not involve that unit. In the present formulation of the SI the basis of the definitions is different from that used previously, so new methods may be used for the practical realization of SI units". In the New SI there are only realizations tout-court based on experimental methods.

Instead, the term "practical" too easily recalls the kind of realizations previously called "mises en pratique", though the latter term is no longer explicitly used in the $2016 \mathrm{draft}$ of the Brochure, except by saying that "descriptions of realizations are also called "mises en pratique"" [4]-this statement is shown later in this section to be incorrect.

Because of that, the last sentence in Section 2.1, stating "The Consultative Committees $[\mathrm{CC}]$ provide the frame for establishing the equivalence of the realizations in order to harmonize the traceability world-wide" [4] can too easily be misunderstood as expressing the need for the CCs to establish new "mises en pratique", as they have done so far.

Thus, the above description is not only extremely confusing, but also incorrect, for two main reasons:

- In the present SI, all realizations different from those indicated in the unit definition (definitional method) were necessarily part of the "mise en pratique" of each base unit. This was then the task of the CCs following the introduction of this category of realizations, which were called "practical" just to distinguish them from the "definitional" ones of each base unit. However, this is no longer the case in the New SI, as amply illustrated hereinbefore. 
- The activity of the BIPM Comités Consultatifs of the various base quantities is presently directed towards preparing the extension of the attribute "mise en pratique" to all the actual realizations of the new units.

However, under the new definition a big question mark remains presently unexplained: can the new realizations still be called mises en pratique according to the present definition of this expression? [15] In fact, the hierarchy [16] between the mises en pratique and the definitional methods vanishes due to the formal lack of the latter methods.

In addition, the different realization methods of the new definition can be subdivided into two categories:

- those called "primary methods" in [4]: "The highest-level experimental methods used for the realization of units using the equations of physics are known as primary methods. The essential characteristic of a primary method is that it allows a quantity to be measured in a particular unit by using only measurements of quantities that do not involve that unit." Actually the second sentence is unnecessary: it is sufficient to say that they are 'primary' because their model includes the relevant constant(s) among the influence quantities ("using the equations of physics", e.g., the watt balance for the kilogram, acoustic gas thermometry for the kelvin), i.e., having the constant in their equation. They were the same methods used for determining the numerical values of the constants: however, in a 'realization' they will be used by assigning to the constant(s) the stipulated value(s);

- those that cannot be labelled 'primary' because they can only indirectly be related (traceable) to the realization of constants. Only this type might remain labelled realizations of a "mise en pratique", providing indirect consistency with the relevant constant(s) (e.g., with the aid of the lattice spacing of a single crystal of highly enriched ${ }^{28} \mathrm{Si}$ for the kilogram; the ITS-90 Scale for the kelvin).

Do both categories (1) and (2) have the same status of realization methods? Probably yes. Can all still be called "mise en pratique"? Probably not - in all instances, they should all be promulgated by the CIPM, like the mises en pratique, not by the CGPM.

Without definitional methods, and without actual official explanation about how this can be worked out, one has to assume that any procedure is applicable for any method that is demonstrated to have the capability to provide a numerical value compatible with that stipulated for the relevant constant(s), meaning that it is "metrologically compatible" [6] with the one(s) stipulated, i.e. whose uncertainty interval contains the stipulated value. The uncertainty interval does not need to have the narrowest width of the top experiments, but must be compatible with an uncertainty sufficient to that country.

It means that one has to understand that the lack of a definitional method does not mean full freedom in choosing a method, because not all will comply with the new definition. The methods and their procedures, to be compliant, need:

- to be traceable to the relevant constant(s);

- to "realise the stipulated value", i.e. the correct magnitude of the unit (within the non-uniqueness of the methods).
Table 1 summarises the metrological ladder. The column "Present SI" is taken from [16].

Hints to cast light on this issue are not presently found in official documents: the documents should instead clarify, at least, that:

- this should mean that not all methods presently known are potentially able to realise a unit in a way that is necessarily fit for an intended use;

- there should be a conceptual difference between those methods that explicitly contain, among their model quantities, the relevant constant(s) (the type reported in step -2 of Tab. 1), and the methods that do not, but that are traceable to the constant(s)' value(s) (the type reported in step -3 of Tab. 1);

- the two types above should not be confounded in a single set, because only the methods in step -2 can be considered equivalent to a definitional method, while only those in step -3 constitute a mise en pratique under the current meaning [3];

- methods in step -2 of Table 1 can only be those that are considered-or are presently used-for obtaining a numerical value of the constant(s).

This fact changes in many respects the duties of the National Metrology Institutes (NMI) wanting to realise independently their own National standards of the base units, as discussed extensively in [16]. Basically, since at the top of the metrological traceability pyramid there are (only) the very definitions of the units, this would require each NMI to provide its own determination of all the relevant constants, as the only means to demonstrate that its chain of standards comply with the condition set by the definitions.

However, should the new definitions become "immaterial", how could a transfer standard be available that could be "calibrated" at (B) and used at (A)? Certainly not under the definition of "calibration" in VIM clause 2.39 in [6]. The only solution might seem to rely on one of the "old fashioned' standards included in the previous mise en pratique. However, as already pointed out, the issue here is not necessarily about getting a smaller uncertainty, but the fact that what is transferred by (B) to (A) would not comply with the very definition of the unit, but would be only a proxy. The very definition of the constant-based units looks to be possibly not able to be propagated among laboratories. Are people to be expected to explain what could be a mass standard "calibrated" in order to ensure metrological traceability to exactly the value $6.626070040 \times 10^{-34} \mathrm{~J} \mathrm{~s}$ with an uncertainty of $10^{-41} \mathrm{~J} \mathrm{~s}$ ? Or, for a unit requiring a scale, how a calibrated thermometer could be considered traceable to a stipulated numerical value of the Boltzmann constant - and, at the same time, of the Planck constant and of $\Delta v_{\mathrm{Cs}}$ ?

\subsubsection{The hierarchical issue in the Metre Convention and in the SI:2018 proposed formulation}

The issues in the previous section bring to the forefront another problem, not strictly scientific, but certainly of basic importance in the metrological frame: what will happen to the absence of hierarchy between the NMIs that 
the Metre Convention ensures to those countries choosing to avoid dependence on another country for particular measurement units, namely the base units? The issue is illustrated in [16], where also the different meaning of hierarchy in metrology is summarised in Table 1: in the present paper, hierarchy means that a country does depend on the standards of another country for its primary standards of particular measurement units, more specifically the base units. There is a basic difference in the metrological ladder that establishes the hierarchy among the different realization methods between the present SI and the New SI, summarised here in Table 1.

In the present SI, every country is, in principle, enabled to make its own standards, i.e., to realise "step 0", the realization of the definition by using the definitional method.

This problem was also there from 1983, when the type (c) definition was introduced for length. Actually, the definition (of the EUD type) does not explicitly say that it refers to the fundamental constant $c_{0}$, and in fact it does not specify the quantity "velocity": the number 299792458 is not a speed but refers to a "time interval": thus, in principle, a different stipulation could follow later. These subtleties are important in a definition. However, the current practice, since then, has considered that $c_{0}$ was stipulated and that no more measurements of $c_{0}$ were possible. Actually none are available after that date. In addition, steps 0 and -1 were confused when defining the mise en pratique.

At present, a hierarchy involves the mises en pratique as the second step of the pyramid below the unit definition (Tab. 1). Quoting from [16], using the mise en pratique of the metre as an example: "the mise en pratique lists the very definition of the metre as the allowed method (a) /the definition of the unit]. It is very possible-even, one would say, normal - that a definition can be directly implemented in practice. However, this fact should not be taken as implying that the other methods included in the mise en pratique are hierarchically at the same level of the definition of the unit, the latter being unique".

In the proposed New SI, at the top step of the hierarchical ladder one should, in view of the lack of definitional methods, demonstrate instead compliance with the condition of being able to obtain the value compatible with the stipulated one for each relevant constant (step 0). Then come the primary methods, which are directly traceable to the constants (step -1 ), while the term "mise en pratique" can only apply to standards of step -2 .

In addition, with the proposed new definitions for the base units as they presently stand, it is true that the present standards can still be used, at least initially, without needing any change: they do conform to the new definition. However, the new definition introduces two new steps above the mises en pratique (see Tab. 1), and so still requires each NMI to provide facts to support evidence of metrological traceability up to the level of the new definitions, for any units that an NMI intends to realise nationally. From the point of view of the hierarchy, this means that every country wishing to be classified at the top of the hierarchical ladder for a specific base unit should have implemented one of the methods at step -1 of the ladder (if not step 0 ), for example for mass, the watt balance. In other words, this would imply a mandatory metrological need for future direct realizations of (some of) those constants at NMIlevel [16].

That is a basic change, since only a handful of NMIs in the entire world would be likely to be able to stay at the top of the hierarchy.

This fact has already been raised in [24] as a "loss of accreditation". The issue is probably the most sensitive difficulty to resolve in order to progress toward the widest acceptance of the SI:2018, and needs be clarified.

\section{Conclusions}

The present CCU 2016 draft of the 9th Brochure can be considered an advancement in terms of correctness with respect to the 2013 draft, having incorporated several comments and reasonings consistently suggested in that period of time in published and in submitted papers.

However, there are still many issues that still have not yet been spelled out correctly (or at least not clearly enough), and a great many issues that have been addressed where ambiguity remains.

In general, a reader - even an informed one-finds herself helpless to understand the actual intention of the CCU, and at a loss as to how to read the New SI, especially with respect to the implementation for users. Many of the "illustrations" contained in this Draft (more could have been cited) tend towards doubt. The SI Brochure is stated to be " $a$ CIPM document requested by the CGPM". This means that, should it not be adequately instructive (the CCU also drafts a CGPM Resolution, then approved by the CIPM, to be approved by the CGPM as the final formal decision), one might wonder how the CGPM can discuss an issue for which no informative document is supplied by the BIPM.

In this respect, the intention of this paper was to fill in some of the information gaps, concentrating quite naturally on features and issues (from Sect. 4) that are today still considered to be contentious within part of the metrological community, but that are not necessarily easy to find in the open literature or official documents: for example, what in this paper has been called the two "frames" (constants and base units), whose interrelation and reciprocal hierarchy is far from clear, though this affects one of the basic needs of any user-understanding his duties. To cite another example, a third basic metrological principle - the internal degree of consistency of the units of a set of inter-related quantities -in addition to the lack of definitional methods and to the continuity of the units through the changes in definition, is presently not tackled at all in official documents.

\section{References}

1. http://www.bipm.org/en/measurement-units/history-si/ [20 June 2016]

2. http://www.bipm.org/en/worldwide-metrology/metre-con vention/ [30 May 2016] 
3. http://www.bipm.org/en/measurement-units/, http://www. bipm.org/en/publications/si-brochure/ [30 May 2016]

4. Comité Consultatif des Unités (CCU), New SI, Draft of 9th edition of the SI Brochure, issued on December 11, 2015, in Draft Documents on http://www.bipm.org/en/measure ment-units/new_si/ (January 4, 2016). Replaces CCU, New SI, Draft Chapters 1, 2 and 3 of the 9th SI Brochure, 2013 in Draft Documents on http://www.bipm.org/en/ measurement-units/new_si/ [9 December 2015]

5. http://metrologybytes.net [30 May 2016]

6. BIPM, International vocabulary of metrology-basic and general concepts and associated terms (VIM) (2008), 3rd edn, http://www.bipm.org/en/publications/guides/ [30 May 2016]

7. Commission IUPAC I-1, Quantities, units and symbols in physical chemistry, monograph (RSC, London, 2007), p. 176

8. F. Pavese, P. Ciarlini, Classes of intercomparisons and the case of temperature standards, PTB Berichte, PTB-IT-10, 63-76 (2003); F. Pavese, Dependence of the treatment of systematic error in interlaboratory comparisons on different classes of standards, ACQUAL 15, 305-315 (2010)

9. F. Pavese, Mathematical and statistical tools in metrological measurement, 2013, Chapter in physical methods, instruments and measurements, in Encyclopedia of life support systems (EOLSS), Developed under the Auspices of the UNESCO, edited by UNESCO-EOLSS Joint Committee (Eolss Publishers, Oxford, UK), http://www.eolss.net

10. S.S. Stevens, On the theory of scales of measurement (PDF), Science 103, 677-680 (1946)

11. http://www.bipm.org/en/publications/si-brochure/appen dix1-thermodynamic-temperature.html, CIPM (2005) Resolution 2 [30 May 2016]
12. http://www.bipm.org/en/CGPM/db/13/1/ 13th CIPM (1967/68) Resolution 1 [30 May 2016]

13. I. Johansson, Constancy and Circularity in the SI, on http:// metrologybytes.net

14. P. De Bièvre, What is the meaning of the concept 'consistency' in (chemical) measurement? Accred. Qual. Assur. 18, 157-158 (2013)

15. http://www.bipm.org/en/publications/mises-en-pratique/ [30 May 2016]

16. F. Pavese, How much does the SI, namely the proposed 'new SI', conform the spirit of the Metre Treaty? Accred. Qual. Assur. 19, 307-314 (2014)

17. R. Schwartz, Ph. Richard, C. Ehrlich, Y. Miki, OIMLBulletin-New-SI-Draft-v4-20120831_Draft.doc (2012)

18. Available online: http://www.bipm.org/en/measurementunits/new-si/ (2013) [4 January 2016]

19. CODATA, http://physics.nist.gov/cuu/Constants/index. html [30 May 2016]; http://www.bipm.org/extra/codata/ [30 May 2016]

20. F. Pavese, A critical review of the CCU 2016 Draft of the Brochure for the "New SI" definition using (fundamental) constants (2016), p. 22, arXiv:1601.00857v.3

21. F. Pavese, Rounding and notation, namely when using stipulations in the definition of measurement units, Measurement 46, 3725-3729 (2013), ISSN 0263-2241

22. M. Mari, F. Pavese, A conceptual roadmap for setting up a system of units in the "New SI" context, arXiv: 1604.00982

23. D.B. Newell, A more fundamental International System of Units, Phys. Today 67, 35 (2014), doi:10.1063/PT.3.2448

24. K.A. Bronnikov, V.N. Melnikov, The fundamental constants ant the transition to new definitions of the SI units, Meas. Tech. 53, 1196-1203 (2011)

Cite this article as: Franco Pavese, Abdérafi Charki, Some important features of the proposed new definition of the International System of Units (SI): realization and hierarchical problems that the users should know about, Int. J. Metrol. Qual. Eng. 7, 403 (2016) 